Rev Inv Vet Perú 1999; 10(1): 34-38

\title{
PRESENCIA DE FORMAS PARASITARIAS EN PECES COMERCIALES DEL MAR PERUANO
}

\author{
Ibelice Pérez, Amanda Chávez ${ }^{2}$ y Eva Casas²
}

\section{Abstract}

The ocurrence of Diphyllobothriidae plerocercoids larval and Anisakidae larva infections in five commercial sea fishes Trachurus symmetricus murphyi "jurel", Sarda sarda chiliensis "bonito", Mugil cephalus "lisa", Odontesthes regia regia "pejerrey" and Sciaena deliciosa "lorna"; was evaluated. The specimens were collected at Callao between April and June 1997. The frequency of Diphyllobothrium sp. larvae was 4.29\% (3/70) in Jurel and of Anisakidae larvae was 8.6\% (6/70), 47.1\% (33/70) and 20\% (14/ 70 ) in jurel, bonito and lisa, respectively. The Anisakidae larvae were Anisakis simplex (Anisakis Type I larva), Anisakis physeteris (Anisakis Type II larva), Hysterothylacium aduncum and Contracaecum sp. Other parasite larval identified included Corynosoma obtuscens, Tentacularia coryphaenae, Nybelinia sp. and Proletus sp.

Key words: Diphyllobothrium, Anisakis, fish parasitism, jurel, bonito, lisa, pejerrey, lorna.

\section{Resumen}

Con el objetivo de evaluar infecciones por larvas plerocercoides de Diphyllobothriidae y larvas de Anisakidae, se estudiaron Trachurus symmetricus murphyi "jurel", Sarda sarda chiliensis "bonito", Mugil cephalus "lisa", Odontesthes regia regia "pejerrey" y Sciaena deliciosa "lorna"; correspondiente a cinco especies de peces marinos comerciales, los mismos que fueron colectados del Terminal Pesquero del Callao en Lima-Perú durante abril a junio de 1997. La frecuencia de larvas plerocercoides de Diphyllobothriidae fue 4.29\% (3/70) en jurel y de larvas de Anisakidae fue $8.6 \%$ (6/70), 47.1\% (33/70) y 20\% (14/70) para jurel, bonito y lisa respectivamente. Las larvas de Anisakidae fueron Anisakis simplex (Larva Anisakis tipo I), Anisakis physeteris (larva Anisakis tipo If), Hysterothylacium aduncum y Contracaecum sp. Otras especies larvarias encontradas fueron: Corynosoma obtuscens, Tentacularia coryphaenae, Nybelinia sp. Proleptus sp.

Palabras Clave: Diphyllobothrium, Anisakis, parasitismo en peces, jurel, bonito, lisa, pejerrey, lorna.

\section{Introducción}

Los peces marinos de consumo

1 Práctica privada

2 UNMSM Apdo. 41-0068 Lima-Perú. Lab. de Parasitologia. E.mail:d170032@unmsm.edu.pe humano, en su mayoría teleóstomos, pueden ser portadores de formas larvarias de parásitos que presentan un ciclo biológico principalmente marino. Algunas de estas formas larvarias pueden infectar al hombre y dar lugar al desarrollo del parásito adulto como el caso del Diphylobothrium sp.; céstode de la familia Diphyllobothriidae con 
su forma larvaria denominada plerocercoide, u ocasionar un parasitismo sin desarrollo de la forma adulta, como el caso de los nemátodos de la familia Anisakidae (Atías, 1991). Las infecciones denominadas Difilobotriasis y Anisakiasis son adquiridas al consumir pescados crudos, insuficientemente cocidos, ahumados o sometidos a congelamiento insuficiente, y que contengan las larvas infectivas de estos helmintos. La fuente de infección en la costa sudamericana del Pacífico radica en el consumo de pescado crudo bajo la forma del "cebiche", "suchi" y "sashimi"(Atias, 1992; Acha y Szyfres, 1989).

Durante el período de abril a junio de 1997 se realizó un estudio con el objetivo principal de determinar la frecuencia de larvas plerocercoides de la familia Diphyllobothriidae y larvas de la familia Anisakidae en diferentes especies de peces comerciales de la costa peruana, así como la identificación y frecuencia de otros géneros de helmintos.

\section{Materialy Motodos}

El estudio se realizó en 307 peces marinos de consumo humano y tamaño comercial (Ministerio de Agricultura, 1966). Se evaluaron 70 especímenes por cada especie de Trachurus symmetricus murphyi "jurel", Sarda sarda chiliensis "bonito", Mugil cephalus "lisa", Odontesthes regia regia "pejerrey" y 27 especímenes de Sciaena deliciosa "lorna". En este último caso no pudo completarse el tamaño de la muestra de 70 debido a su desaparición en los mercados ocasionado por el fenómeno "El Niño". Todos los peces muestreados fueron procedentes del Terminal Pesquero del Callao.

Se realizó la apertura y posterior visualización in situ de las vísceras abdominales, peritoneo parietal y la disección de órganos y músculos. Las larvas parasitarias encontradas fueron lavadas en suero fisiológico y fijadas en alcohol etílico al 70\%. Se tomaron medidas de las larvas y quistes con una regla milimétrica y ocular micrométrico. Se utilizaron las claves descritas por Chirichigno (1974) para la identificación de los peces en estudio (nombres científicos y comunes).

La identificación de las larvas plerocercoides de la familia Diphyllobothriidae, así como de las larvas de la familia Anisakidae se basaron en las características morfológicas y dimensiones descritas por Tantaleán (1972) y Cattán y Videla (1976).

\section{Resultados}

En el Cuadro 1 se muestra la frecuencia porcentual de las larvas parásitas de las familias Diphyllobothriidae y Anisakidae, así como formas larvarias de otras familias de parásitos, que fueron encontrados en las cinco especies de peces comerciales.

La forma larvaria de la tenia Diphylobothrium sp.; perteneciente a la familia Diphyllobothriidae denominada plerocercoide, se encontró únicamente en el jurel. Larvas infectivas de Anisakidae se encontraron en jurel, bonito y lisa. Dentro de otras formas larvarias, cuyas formas adultas parasitan en mamíferos marinos y terrestres, se encontró el Corynosoma obtuscens en jurel y lorna, Tentacularia coryphaenae, Nybelinia sp. y Proleptus sp. en el jurel, bonito y lorna.

La localización de las larvas plerocercoides de Diphyllobothridae fue principalmente en la superficie del hígado, las mismas que se ubicaban dentro de quistes 
Cuadro 1. Frecuencia de larvas de parásitos hallados en 70 especímenes de 5 especies de peces marinos comerciales ${ }^{1}$.

\begin{tabular}{|c|c|c|c|c|c|c|c|c|}
\hline \multirow[t]{2}{*}{ Larvas Parásitas } & \multicolumn{2}{|c|}{ Jurel } & \multicolumn{2}{|c|}{ Bonito } & \multicolumn{2}{|c|}{ Lisa } & \multicolumn{2}{|c|}{ Lorna } \\
\hline & $\mathrm{N}^{\circ}$ & $\%$ & $\mathrm{~N}^{\circ}$ & $\%$ & $\mathrm{~N}^{\circ}$ & $\%$ & $\mathrm{~N}^{\circ}$ & $\%$ \\
\hline Fam. Diphyllobothriidae & 3 & 4.3 & - & - & - & - & - & - \\
\hline Fam. Anisakidae & 6 & 8.6 & 41 & 59.2 & 14 & 20 & - & - \\
\hline Anisakis simplex & 5 & 7.1 & - & - & - & - & - & - \\
\hline Anisakis physeteris & 1 & 1.4 & 17 & 24.3 & - & - & - & - \\
\hline Contracaecum sp. & - & - & - & - & 14 & 20 & - & - \\
\hline Hysterothylacium aduncum & - & - & 24 & 34.3 & - & - & - & - \\
\hline Otras especies & 7 & 10.0 & 1 & 1.4 & - & - & 18 & 66.7 \\
\hline Corynosoma obtuscens & 1 & 1.4 & - & - & - & - & 9 & 33.3 \\
\hline Tentacularia coryphaenae & 2 & 2.9 & - & - & - & - & - & - \\
\hline Nybelinia sp. & 4 & 5.7 & 1 & 1.4 & - & - & 2 & 7.4 \\
\hline Proleptus sp. & & - & - & - & - & - & 7 & 26.0 \\
\hline Total & 16 & 22.9 & 42 & 58.6 & 14 & 20 & 18 & 66.7 \\
\hline
\end{tabular}

esféricos de color blanco nacarado que medían 2.1 por $2.2 \mathrm{~mm}$. Las larvas medían $10.7 \mathrm{~mm}$. de longitud y $0.98 \mathrm{~mm}$. de ancho en promedio, siendo la longitud del scolex de $0.9 \mathrm{~mm}$.

La localización de las larvas de Anisakidae fue principalmente en el intestino delgado en el jurel y bonito, y en el hígado en el caso de la lisa. Las formas larvarias de Anisakis sp. y Contracaecum sp. fueron halladas en su mayoría encapsuladas por tejido fibroso; mientras que, para el caso de Hysterothylacium aduncum, fueron halladas libres en las superficie de los órganos abdominales. Las medidas de las larvas de Anisakidae variaron de 10.3 a 26.5 $\mathrm{mm}$ de longitud por 177.1 a $906.6 \mu \mathrm{m}$ de ancho y las medidas de sus quistes fueron de 3.3 a $5.1 \mathrm{~mm}$ por 3.3 a $4.1 \mathrm{~mm}$.

El total de peces marinos comerciales estudiados que se encontraban parasitados fue de 22.9 en jurel, 58.6 en bonito, 20.0 en lisa y $66.7 \%$ en lorna. No se encontraron larvas de parásitos helmintos en cavidad abdominal ni en músculo del pejerrey.

\section{Discusión}

La presencia de formas parasitarias está influida por muchos factores que puede ir desde el tamaño del pez (al ser estos parásitos acumulativos, a mayor tamaño del pez, existe mayor probabilidad de encontrar larvas de parásitos), hasta cambios en la ecología marina como temperatura, luz, disminución de huéspedes intermediarios, hábitat, etc.

El hábitat presenta también diferentes temperaturas, así se tiene que, la temperatura normal que registra el mar de Perú, en la zona costera es de 14 a $15^{\circ} \mathrm{C}$ y mar adentro 20 a $26^{\circ} \mathrm{C}$., y en los meses de verano registra un ingreso de las aguas calientes hacia la costa. El nivel de oxígeno es importante para el desarrollo de la vida marina, niveles de $1 \mathrm{ml} / 1$ ocasiona la muerte, con $2 \mathrm{ml} / \mathrm{l}$ se presenta dificultad y en ocasiones el no desarrollo. A $50 \mathrm{~m}$. de profundidad en nivel de oxígeno es de $1 \mathrm{ml} / 1$ o menos. Durante el fenómeno "El Niño" el nivel de oxígeno aumenta en zonas donde normalmente es de cero a $2 \mathrm{ml} / \mathrm{l}$ y la 
temperatura aumenta considerablemente, lo cual puede favorecer el desarrollo de otras formas de vida y de ciclos biológicos.

El parasitismo está íntimamente relacionado a los hábitos alimenticios de los peces. El bonito, jurel y lorna son peces de vida pelágica, mientras que la lisa y el pejerrey de vida demersal, esto significa que las tres primeras especies viven en aguas libres, mar adentro y basan su alimentación en pequeños peces, no obstante la lorna también puede vivir en fondos arenosos en las cercanías de las playas. La lisa y el pejerrey viven cerca de las playas en fondos arenosos y de poca profundidad, alimentándose en caso de la lisa de diatomeas, algas finas y barro, mientras que el pejerrey basa su alimentación en organismos planctónicos. Todo esto permite el surgimiento de diferentes hábitats, hospederos y ecosistemas para el establecimiento de diversos ciclos parasitarios marinos de las especies descritas. (Tantaleán, 1975; Ministerio de Agricultura, 1966).

En el presente trabajo se obtuvo una frecuencia de $4.3 \%$ de larvas plerocercoides de la familia Diphyllobothriidae en el jurel no encontrándose dicha forma larvaria en las restantes especies. En un estudio similar evaluando lorna, coco, cojinova, jurel y lisa, durante el período 1970-1971, con peces provenientes del terminal pesquero del Callao y el área marítima de Chimbote, Tantaleán (1975) halló una frecuencia de $6.5 \%$ para lorna y de $0.5 \%$ para coco. En este estudio no ha sido posible encontrar larvas plerocercoides de Diphyllobothriidae en bonito y lorna. Sin embargo estos peces han sido descritos junto al jurel como hospederos paraténicos de Diphyllobothrium pacificum en el mar del Perú (Tantaleán y Huiza, 1994).

Los hallazgos de larvas de anisakidae encontrados en jurel en el presente estudio son inferiores $(8.6 \%)$ al obtenido por Tantaleán (1972) quien trabajando con jurel, coco, y lorna obtuvo una frecuencia de 48.6, 1.6 y $1.5 \%$, respectivamente. Los factores que hayan podido influir en estos resultados son semejantes a los descritos para la familia Diphyllobothriidae. El no hallazgo de larvas Anisakidae en la lorna, pudo deberse a la reducción en tamaño muestral, de 70 peces estimados al inicio del trabajo a 27 peces. La lisa es descrita como hospedero de Contracaecum sp. por Morales (1997) quien reporta una prevalencia de $29.9 \%$ en muestreos llevado a cabo entre 1982 a 1983 en la Bahía de Paracas; el presente estudio reporta resultados similares (20\%). En el bonito se halló una frecuencia alta para Hysterothylacium aduncum de $34.3 \%$, no encontrándose referencias en la literatura que reporten este hallazgo en ese pez.

En el caso de jurel, bonito y lorna se sabe que se alimentan de peces pequeños, lo cual explicaría la presencia de formas larvarias. En lo referente a la lisa por encontrarse como portadora de Contracaecum sp. se deduce que puede incluir en su alimentación invertebrados (crustáceos), puesto que el consumo de algas no juega ningún papel en el ciclo biológico de este parásito. En el caso del pejerrey su tipo de alimentación permitiría explicar la razón de no ser reportado como hospedero de estas formas larvarias.

Otras larvas encontradas en este estudio y cuyas forma adultas parasitan mamíferos marinos y terrestres son el Corynosoma obtuscens, que puede parasitar caninos (Rojas, 1997), hallado en jurel y lorna, en 1.4 (1/6) y 33.3\% (9/14), respectivamente. Tentacularia coryphaenae, Nybelinia sp. y Proleptus sp. hallados en diferentes frecuencias en el jurel, bonito y lorna (Cuadro 1), y tienen como hospederos definitivos a tiburones y rayas (Tantaleán y 
Huiza, 1994).

Todos los factores descritos en un inicio pueden explicar una mayor variedad de parasitismo en los peces que viven en altamar (jurel, bonito y lorna), mientras que la lisa presentó un parásito y el pejerrey ninguno.

\section{Ditomtura Citada}

1. Acha, N.P. y B. Szfres. 1989. Zoonosis y enfermedades transmitibles comunes al hombre y los animales. $2^{\text {da }}$ ed. Washington:OPS 721-726, 784-789.

2. Atias, A. 1991. Parasitología Clínica. In Carbajal J. Anisakiasis; Torres y Pérez C. eds. Difilobotriasis $3^{\text {th }}$ ed. Chile: Mediterráneo. 190-193, 203-212.

3. Cattan, P.E. y N.N. Videla. 1976. Presencia de larvas de Anisakis sp. en el Jurel Trachurus murphyi Nichols, 1920 (algunas consideraciones con el granuloma eosinofílico en el hombre). Bol. Chile. Parasit. 31:71-74.

4. Chirichigno, F.N. 1974. Clave para identificar los peces marinos del Perú. Informe $N^{\circ} 44$ IMARPE. Callao-Perú.

5. Ministerio de Agricultura - Servicio de Pesquería. 1966. Peces comunes de la Costa Peruana. Serie de divulgación científica $\mathrm{N}^{\circ} 6.2^{\text {th }}$ de Perú.; 4-5, 12, 39, 54-56.
6. Morales, M., R. Severino, H. Ternoche, S. Sampertegui y G. Guillén. 1997. Parasitofauna de la Reserva Nacional de Paracas VII, parásitos de alevinos de Mugil cephalus "liza"y su relación con el fenómeno "El Niño". II Congreso Peruano de Parasitología, ArequipaPerú. Setiembre 17-19: 105.

7. Rojas, R., M. Dávalos y R. Cabrera. 1997. Prevalencia de Corynosoma obtuscens (Acanthocephala) en Canis familiaris de la provincia de Chincha y Pisco 1996, II Congreso Peruano de Parasitología, Arequipa - Perú. Setiembre 17-19: 82.

8. Tantaleán, V.M. 1975. Hallazgo de larvas plerocercoides de Diphyllobothriidae en peces del mar peruano. Bol. Chile Parasit. 30: 18-20.

9. Tantaleán, V.M. y F.A. Huiza. 1994. Sinopsis de los parásitos marinos de la costa peruana. Biotempo. 1: 53-101.

10. Tantaleán, V.M. 1972. La presencia de larvas de Anisakis sp. en algunos peces comerciales del mar peruano. Rev. Per. Med. Trop., U.N.M.S.M. 11(1): 38-43.

11. Tantaleán, V.M. y F.A. Huiza. 1993. Nematode larvae with medical importance found in sea fish from the Peruvian shore, with two records of human infections. Rev. Per. Med. Trop. U.N.M.S.M. 7(1): 61-65. 